\title{
First-principles approach to the charge-transport characteristics of monolayer molecular-electronics devices: Application to hexanedithiolate devices
}

\author{
Yong-Hoon Kim, ${ }^{1, *}$ Jamil Tahir-Kheli, ${ }^{2}$ Peter A. Schultz, ${ }^{3}$ and William A. Goddard III ${ }^{2, \dagger}$ \\ ${ }^{1}$ Korea Institute for Advanced Study, 207-43 Cheongnyangni 2-dong, Dongdaemun-gu, Seoul, 130-722 Korea \\ ${ }^{2}$ Materials and Process Simulation Center, California Institute of Technology, Pasadena, California 91125-7400, USA \\ ${ }^{3}$ Multiscale Computational Materials Methods Department, Sandia National Laboratories, Albuquerque, New Mexico 87185, USA
}

(Received 5 December 2005; revised manuscript received 10 April 2006; published 21 June 2006)

\begin{abstract}
We report on the development of an accurate first-principles computational scheme for the charge transport characteristics of molecular monolayer junctions and its application to hexanedithiolate (C6DT) devices. Starting from the Gaussian basis set density-functional calculations of a junction model in the slab geometry and corresponding two bulk electrodes, we obtain the transmission function using the matrix Green's function method and analyze the nature of transmission channels via atomic projected density of states. Within the developed formalism, by treating isolated molecules with the supercell approach, we can investigate the current-voltage characteristics of single and parallel molecular wires in a consistent manner. For the case of single C6DT molecules stretched between $\mathrm{Au}(111)$ electrodes, we obtain reasonable quantitative agreement of computed conductance with a recent scanning tunneling microscope experiment result. Comparing the charge transport properties of C6DT single molecules and their monolayer counterparts in the stretched and tilted geometries, we find that the effect of intermolecular coupling and molecule tilting on the charge transport characteristics is negligible in these devices. We contrast this behavior to that of the $\pi$-conjugated biphenyldithiolate devices we have previously considered and discuss the relative importance of molecular cores and molecule-electrode contacts for the charge transport in those devices.
\end{abstract}

DOI: 10.1103/PhysRevB.73.235419

PACS number(s): 73.63. $-\mathrm{b}, 85.35 .-\mathrm{p}, 85.65 .+\mathrm{h}$

\section{INTRODUCTION}

In the rapidly developing field of molecular electronics, ${ }^{1,2}$ an important strategy of device fabrication is forming a selfassembled monolayer (SAM) or placing a LangmuirBlodgett (LB) monolayer on an electrode and attaching the second electrode in such a way as to form wires, diodes, switches, etc. ${ }^{3-5}$ Due to the intermolecular interactions, one can possibly achieve better characterized molecular structures and correspondingly more robust device control mechanisms within the monolayer than with individual molecules. Upon formation of a monolayer, electronic energy band formations as well as conformational modifications might result in changes in the device charge transport characteristics. Experimental studies addressing the effect of monolayer formation in saturated and $\pi$-conjugated molecules on the device properties are now beginning to appear. ${ }^{6-8}$ First-principles theoretical studies will be very valuable in this effort by supplementing and guiding the experiments.

For this purpose, we have developed a scheme to calculate the current-voltage $(I-V)$ characteristics of a monolayer molecular device. It is based on the Landauer-Büttiker formalism that relates the current with the transmission function, and compute the transmission function through the matrix Green's function (MGF) method applied to the linearcombination of atomic orbitals (LCAO) density-functional theory (DFT) calculations. Currently, the code is coupled with a Gaussian basis set code, ${ }^{9}$ but it can be easily generalized to other LCAO DFT or tight-binding codes. This method has been successfully applied to the investigation of molecular electronic devices based on monolayers of simple biphenyldithiolate ${ }^{10}$ (BPDT) and complex catenane molecules. ${ }^{11}$
The MGF approach has been previously implemented on the basis of zero-dimensional (molecular) quantum chemistry codes ${ }^{12-15}$ and periodic solid state codes, ${ }^{16-20}$ and applied to the study of molecular electronic devices. However, to our knowledge, the effect of intermolecular interactions on the device charge transport characteristics within the rigorous two-dimensional (2D) monolayer geometry has not yet been considered. The unique strength of our scheme is that the 2D periodicity of monolayer devices and the semi-infinite nature of electrodes are treated in a consistent and accurate manner by the reciprocal-space k-point sampling. Applying this method, we consider in this work the charge transport characteristics of devices based on a single hexanedithiolate (C6DT) molecule and its monolayer counterparts in the stretched and relaxed molecular geometries. We show that the role of intermolecular interactions as well as the molecule tilting on the charge transport characteristics is minimal for the C6DT molecules. We trace the origin of this robust electronic transport behavior to the energetic position of saturated C6 molecular core levels located far from the Fermi level. We contrast this with the observation in $\pi$-conjugated BPDT molecules. ${ }^{10}$ Unlike the case of C6DT molecules where the transmission channel is essentially determined by the DT bridges and the C6 core plays a secondary role, in the case of BPDT molecules, BP core levels are significantly hybridized with DT bridge levels which results in a large variation of conductance with the molecule tilting and monolayer packing. We note that in both cases DT bridge levels play an important role in characterizing the device transmission properties and conclude that the characteristics of the devices based on small molecules strongly depend on the nature of metal-electrode contacts.

The current article is organized as follows. In Sec. II, we first describe our approach for the calculation of $I-V$ charac- 
teristics and relevant implementation details. Accurate estimation of the self-energies and the proper setup of device models will be emphasized. Approximations involved in the current implementation will be discussed. In Sec. III, we study the conformations and charge transport properties of a single C6DT molecule sandwiched between $\mathrm{Au}(111)$ electrodes and its monolayer counterparts. For a stretched single C6DT molecule, we obtain a good quantitative agreement of the conductance with a recent scanning tunneling microscope (STM) experiment. ${ }^{21}$ We next compare the conductivity of single and packed molecules in stretched and tilted geometries and estimate the effect of coupling between molecular backbones on the device charge transport characteristics. In Sec. IV, we will conclude the paper by discussing differences between the saturated C6DT and $\pi$-conjugated BPDT molecules. We point out that observed discrepancies between $n$-alkanedithiolate ${ }^{21,22}$ and monophenyldi(methane)thiolate ${ }^{23}$ experiments can be explained by our findings.

\section{THEORETICAL FORMULATION AND COMPUTATIONAL IMPLEMENTATION}

\section{A. General formalism}

We obtain the $I-V$ characteristics of molecular-scale devices by invoking the Landauer-Büttiker formalism, ${ }^{24}$ which relates the linear response conductance to the transmission probability $T$ :

$$
I(V)=\frac{2 e}{h} \int_{\mu_{1}}^{\mu_{2}} d E T(E, V)\left[f\left(E-\mu_{1}\right)-f\left(E-\mu_{2}\right)\right],
$$

where $\mu_{1}$ and $\mu_{2}$ are the chemical potentials of the bottom and top electrodes, $f(E-\mu)=1 /\left\{1+\exp \left[(E-\mu) /\left(k_{B} T\right)\right]\right\}$ is the Fermi-Dirac distribution function, $k_{B}$ is the Boltzmann constant, and $T$ is the temperature. We assume the zero temperature conductance $T=0$. We also neglect noncoherent charge transport processes that can couple different transverse modes. Thus, the computation of the $I-V$ characteristics amounts to evaluation of the transmission function through independent $\mathbf{k}_{\|}$(surface-parallel direction reciprocal lattice vector point) channels and their integral over the $2 \mathrm{D}$ reciprocal unit cell $\widetilde{\Omega}$,

$$
T(E, V)=\frac{1}{\Omega} \int_{\widetilde{\Omega}} d \mathbf{k}_{\|} T^{\mathbf{k}_{\|}}(E, V),
$$

where $\Omega$ is the area of the reference unit cell surface.

To compute $T^{\mathbf{k}_{\|}}$, we adopt the matrix version of nonequilibrium Green's function (NEGF) approach. ${ }^{24}$ The NEGF theory is a well-developed general formalism to treat various nonequilibrium charge transport phenomena. ${ }^{25}$ In addition, the MGF approach provides a straightforward and efficient way to treat the open boundary problem within the LCAO formalism. ${ }^{26}$ Let us assume that we carried out a LCAO DFT calculation for the device model including semi-infinite electrodes [Fig. 1(a)]. Next, we partition the DFT Hamiltonian matrix $H$ into the LCAO blocks belonging to electrodes 1 and 2 and the molecule in between for each $\mathbf{k}_{\|}$point:

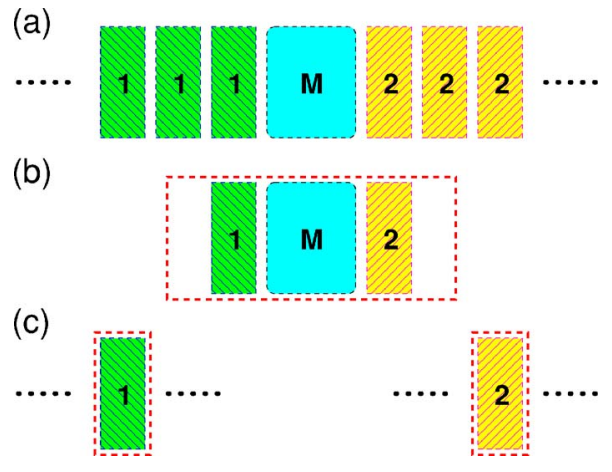

FIG. 1. (Color online) Schematic illustration of the device modeling. (a) The device is constructed by sandwiching a molecular monolayer between electrode 1 and electrode 2 . It is semi-infinite along the electrode surface normal direction. The resulting infinite problem setup is reduced into a finite one via the MGF formulation. Independent DFT calculations are performed for (b) the finite 2D device model including the molecules and part of electrode atoms (represented by the dashed lines), and (c) two 3D bulk cells corresponding to the electrodes 1 and 2 (represented by dashed lines). These three DFT outputs are combined via the MGF method to extract the information on the semi-infinite device. Dots in (a) and (c) denote the periodicity along the surface normal direction. Periodicity along the surface parallel direction is implicitly assumed.

$$
H^{\mathbf{k}_{\|}}=\left(\begin{array}{ccc}
H_{1}^{\mathbf{k}_{\|}} & H_{M 1}^{\mathbf{k}_{\|}^{\dagger}} & 0 \\
H_{M 1}^{\mathbf{k}_{\|}} & H_{M}^{\mathbf{k}_{\|}} & H_{M 2}^{\mathbf{k}_{\|}} \\
0 & H_{M 2}^{\mathbf{k}_{\|}^{\dagger}} & H_{2}^{\mathbf{k}_{\|}}
\end{array}\right) .
$$

Here, we assumed the molecule region is large enough to neglect coupling between the two electrodes.

Partitioning the overlap matrix $S$ in the same manner, the one-particle retarded Green's function $G(E)=(\varepsilon S-H)^{-1}(\varepsilon$ $\left.=E+i \delta, \delta \rightarrow 0^{+}\right)$is written in the matrix form as

$$
\begin{aligned}
G^{\mathbf{k}_{\|}} & =\left(\begin{array}{ccc}
G_{1}^{\mathbf{k}_{\|}} & & \cdots \\
& G_{M}^{\mathbf{k}_{\|}} & \\
\vdots & & G_{2}^{\mathbf{k}_{\|}}
\end{array}\right) \\
& =\left(\begin{array}{ccc}
\varepsilon S_{1}^{\mathbf{k}_{\|}}-H_{1}^{\mathbf{k}_{\|}} & X_{1}^{\mathbf{k}_{\|} \dagger} & 0 \\
X_{1}^{\mathbf{k}_{\|}} & \varepsilon S_{M}^{\mathbf{k}_{\|}}-H_{M}^{\mathbf{k}_{\|}} & X_{2}^{\mathbf{k}_{\|}} \\
0 & X_{2}^{\mathbf{k}_{\|} \dagger} & \varepsilon S_{2}^{\mathbf{k}_{\|}}-H_{2}^{\mathbf{k}_{\|}}
\end{array}\right)^{-1},
\end{aligned}
$$

where $X_{a}=\varepsilon S_{M a}^{\mathbf{k}_{\|}}-H_{M a}^{\mathbf{k}_{\|}}(a=1,2)$. Applying the standard matrix manipulation, the molecule part of $G$ is then obtained as

$$
G_{M}^{\mathbf{k}_{\|}}=\left(E S_{M}^{\mathbf{k}_{\|}}-H_{M}^{\mathbf{k}_{\|}}-\Sigma_{1}^{\mathbf{k}_{\|}}-\Sigma_{2}^{\mathbf{k}_{\|}}\right)^{-1},
$$

where $\Sigma_{a}$ is the non-Hermitian self-energy matrix

$$
\Sigma_{a}^{\mathbf{k}_{\|}}=X_{a}^{\mathbf{k}_{\|}} G_{a}^{\mathbf{k}_{\|}} X_{a}^{\mathbf{k}_{\|} \dagger} \quad(a=1,2) .
$$

The Hermitian part if $\Sigma_{a}$ describes the shift of molecular energy levels and its anti-Hermitian component

$$
\Gamma_{a}^{\mathbf{k}_{\|}}=i\left(\Sigma_{a}^{\mathbf{k}_{\|}}-\Sigma_{a}^{\mathbf{k}_{\| \dagger}^{\dagger}}\right)
$$

represents the broadening of molecular energy levels (inverse lifetime) due to the coupling of molecules to electrodes. Since the self-energy effectively gives rise to a finite 
imaginary term, we may set $\delta=0 .{ }^{24}$ Note that, although the dimensions of $X_{a}$ and $G_{a}$ matrices are still infinite, the dimension of $\Sigma_{a}$ is that of the molecular part, so we have reduced the original infinite problem into a finite one. Related to this point, it should be mentioned that, in practice, the DFT calculation is only passible for a finite model system. We will denote the part of contact matrix $X_{a}$ that is actually calculated as $x_{a}$.

Once $\Gamma_{a}$ and $G_{M}$ are available for a selected set of $\mathbf{k}_{\|}$ points, the transmission functions through the $\mathbf{k}_{\|}$points are computed as ${ }^{24}$

$$
T^{\mathbf{k}_{\|}}=\operatorname{Tr}\left(\Gamma_{1}^{\mathbf{k}_{\|}} G_{M}^{\mathbf{k}_{\|}} \Gamma_{2}^{\mathbf{k}_{\|}} G_{M}^{\mathbf{k}_{M}^{\dagger}}\right),
$$

and the total transmission can be obtained from Eq. (2). Note that Eq. (8) is valid only for the noninteracting electron case. $^{24,27}$

\section{B. Self-energies and surface Green's functions}

The self-energy $\Sigma_{a}$ [Eq. (6)] plays an essential role in the MGF formulation by reducing the original intractable semiinfinite problem into a manageable finite one, and enabling reliable estimation of $I-V$ characteristics. To obtain $\Sigma_{a}$, we need to properly determine the molecule-electrode interaction $X_{a}$ and the electrode-part Green's functions $G_{a}$, and combine them into $\Sigma_{a}$ according to Eq. (8). Especially, because $X_{a}$ and $G_{a}$ are still of semi-infinite-dimension, a procedure to reduce them into finite-dimensional entities is required. First, reliable description of the molecule-electrode contact $X_{a}$ can be achieved by including a large number of electrode atoms in the finite main device model, or by choosing $x_{a}$ that is as large as possible [Fig. 1(b)]. Via including a large number of electrode-region atoms in the main device model, we can accurately determine the charge transfer at the interfaces between the molecule and metal electrodes and the resulting lineup of molecular energy levels with respect to the Fermi energy of the entire system $E_{F} .{ }^{14}$ This is one of the most crucial properties in determining the charge transport characteristics of devices. We will discuss this point further in Sec. II C 1.

Second, for the semi-infinite $G_{a}$, we extracted the surface Green's functions $g_{S a}$ of a finite dimension from two separate three-dimensional (3D) bulk calculations with the unit cells corresponding to the bottom and top electrodes [Fig. 1(c)]. Due to the crystalline periodicity of the bulk, removing one layer of the contact lattice recursively gives back the same surface Green's function (we consider the case of the electrode 2; the electrode 1 case is obtained in a similar manner)

$$
G_{2}^{\mathbf{k}_{\|}}=\left(\varepsilon S_{2}^{\mathbf{k}_{\|}}-H_{2}^{\mathbf{k}_{\|}}\right)^{-1}=\left(\begin{array}{cccc}
g_{S 2}^{\mathbf{k}_{\|}} & & & \\
& g_{S 2}^{\mathbf{k}_{\|}} & & \\
& & g_{S 2}^{\mathbf{k}_{\|}} & \\
\vdots & & & \ddots
\end{array}\right),
$$

where each $g_{S 2}$ represents the LCAO block of matrix elements corresponding to the electrode unit cell.

Our strategy to calculate $g_{S 2}^{\mathbf{k}_{\|}}$is as follows. We first perform two 3D DFT calculations for each bulk electrode. Next, as the resulting $H$ and $S$ matrices are represented in 3D $\mathbf{k}$ space in our DFT calculations, we arrange the $\mathbf{k}$ vectors into the surface-parallel $\mathbf{k}_{\|}$and surface-normal $\mathbf{k}_{\perp}$ components. For the simplicity of implementation, we do not impose any symmetry reduction in selecting $\mathbf{k}$ points except the timereversal symmetry. We also set the $\mathbf{k}_{\|}$points of the electrode DFT calculation [Fig. 1(c)] to be exactly identical to those of the main device DFT calculation [Fig. 1(b)]. Then, for each $\mathbf{k}_{\|}$point, we carry out a one-dimensional Fourier transform of $H$ and $S$ along the surface normal direction to obtain

$$
\begin{aligned}
H^{\left(\mathbf{k}_{\|}, \mathbf{T}_{m}\right)} & =\frac{1}{N_{\mathbf{k}_{\perp}}} \sum_{\mathbf{k}_{\perp}} e^{-i \mathbf{k}_{\perp} \cdot \mathbf{T}_{m}} H^{\left(\mathbf{k}_{\|}, \mathbf{k}_{\perp}\right)}, \\
S^{\left(\mathbf{k}_{\|}, \mathbf{T}_{m}\right)} & =\frac{1}{N_{\mathbf{k}_{\perp}}} \sum_{\mathbf{k}_{\perp}} e^{-i \mathbf{k}_{\perp} \cdot \mathbf{T}_{m}} S^{\left(\mathbf{k}_{\|}, \mathbf{k}_{\perp}\right)},
\end{aligned}
$$

where $\mathbf{T}_{m}$ is the surface-normal direction lattice vector of layer $m$, and $N_{\mathbf{k}_{\perp}}$ is the number of $\mathbf{k}_{\perp}$ point sampled along the surface normal direction. Assuming that the verticaldirection lattice constant of the electrode bulk unit cell is large enough so that only the in-plane $(m=0)$ and the nearest-neighbor-plane coupling $(m=+1)$ matrix elements are nonzero,

$$
\varepsilon S_{2}^{\mathbf{k}_{\|}}-H_{2}^{\mathbf{k}_{\|}}=\left(\begin{array}{cccc}
\alpha_{2}^{\mathbf{k}_{\|}} & \beta_{2}^{\mathbf{k}_{\|}} & 0 & \cdots \\
\beta_{2}^{\mathbf{k}_{\|}^{\dagger}} & \alpha_{2}^{\mathbf{k}_{\|}} & \beta_{2}^{\mathbf{k}_{\|}} & \\
0 & \beta_{2}^{\mathbf{k}_{\| \dagger}^{\dagger \dagger}} & \alpha_{2}^{\mathbf{k}_{\|}} & \\
\vdots & & & \ddots
\end{array}\right),
$$

where

$$
\begin{aligned}
& \alpha_{2}^{\mathbf{k}_{\|}}=\varepsilon S_{2}^{\left(\mathbf{k}_{\|}, \mathbf{T}_{0}\right)}-H_{2}^{\left(\mathbf{k}_{\|}, \mathbf{T}_{0}\right)}, \\
& \beta_{2}^{\mathbf{k}_{\|}}=\varepsilon S_{2}^{\left(\mathbf{k}_{\|}, \mathbf{T}_{1}\right)}-H_{2}^{\left(\mathbf{k}_{\|}, \mathbf{T}_{1}\right)},
\end{aligned}
$$

we apply the same matrix manipulation used to derive Eqs. (5) and (6) from the matrix of Eq. (4) and obtain the recursion relation for the surface Green's function matrix

$$
g_{S 2}^{\mathbf{k}_{\|}}=\left(\alpha_{2}^{\mathbf{k}_{\|}}-\beta_{2}^{\mathbf{k}_{\|}} g_{S 2}^{\mathbf{k}_{\|}} \beta_{2}^{\mathbf{k}_{1} \dagger}\right)^{-1},
$$

which can be solved iteratively.

As a summary, we recapitulate the computation procedure. We start with a 2D DFT calculation for the device model [Fig. 1(b)] and two 3D DFT calculations for the corresponding bulk electrodes [Fig. 1(c)]. The order of the matrix elements in these initial DFT calculations needs not be that of Eq. (3) or (4), hence, we need to reorder the elements of the device DFT $H$ and $S$ matrices into the form of Eq. (3). For the electrode part, we prepare the 2D $H$ and $S$ matrices [Eq. (12)], and for each $E$ calculate $g_{S}$ by solving Eq. (13). We also reorder the matrix elements of $g_{S}$ to be commensurate with the electrode part of Eq. (3) or (4). Having obtained $x_{a}$ and $g_{S}$, we align the Fermi levels of the device model [Fig. 1(b)] and electrodes [Fig. 1(c)], and construct $\Sigma$ for each sampling energy point $E$,

$$
\Sigma_{a}^{\mathbf{k}_{\|}}=x_{a}^{\mathbf{k}_{\|}} g_{S}^{\mathbf{k}_{\|}} x_{a}^{\mathbf{k}_{\|} \|^{\dagger}} .
$$

We finally compute the Green's function according to Eq. (5), with which we compute the transmission function as in 
Eq. (8). The nature of the transmission channels were analyzed via atomic projection of density of states

$$
\operatorname{DOS}(E)=\frac{1}{2 \pi \Omega} \int_{\widetilde{\Omega}} d \mathbf{k}_{\|} \operatorname{Tr}\left[A_{M}^{\mathbf{k}_{\|}}(E) S_{M}^{\mathbf{k}_{\|}}\right],
$$

where $A=i\left(G-G^{\dagger}\right)$ is the spectral function.

\section{Issues: Numerical and theoretical accuracy of the calculation}

We emphasize that Eq. (8) is formally valid only for the noninteracting case, ${ }^{25,27}$ the simplification of which makes the NEGF formalism a popular choice of computational implementation. We obtained the single-particle Hamiltonian within the Perdew-Burke-Ernzerhof (PBE) parametrization of generalized gradient approximation (GGA) ${ }^{28}$ of the KohnSham (KS) DFT formalism. ${ }^{29}$ We here assess the implication of this approximation in terms of numerical and theoretical aspects.

\section{Alignment of Fermi levels and the energetic position of molecular levels}

In the case of adopting a molecular DFT code, or modeling electrodes by clusters, a finite highest-occupied molecular orbital (HOMO)-lowest unoccupied molecular orbital (LUMO) gap is produced in the calculation. In comparison, we perform the DFT calculations of bulk electrodes [Fig. 1(c)] using a periodic DFT code, so we can accurately reproduce the semi-infinite nature of metal electrodes. More explicitly, we can increase the number of $\mathbf{k}$ points sampled in our 3D bulk electrode DFT calculations such that the "numerical" gap converges correctly to the "physical" gap of zero (typically by $<10^{-3} \mathrm{eV}$ ).

In addition, modeling devices within the cluster geometry also results in a finite HOMO-LUMO gap, which forces the Fermi levels of device model and metal electrode models aligned in some arbitrary fashion. In our calculations, we include a large number of metal electrode atoms in the device model [Fig. 1(b)] in the $2 \mathrm{D}$ slab geometry so that the zero "numerical" gap $\left(<10^{-3} \mathrm{eV}\right)$ is also produced in the device model DFT calculation. These ensure that the Fermi levels of the three DFT calculations are aligned unambiguously [Eq. (14)] and the location of molecular levels with respect to $E_{F}$ and their broadening are accurately determined. This represents an important advantage of employing a periodic DFT code rather than a cluster DFT code for the MGF implementation.

\section{Kohn-Sham Hamiltonian and bias dependence}

Even after the numerical accuracy is guaranteed as described in Sec. II C 1, one still needs to exercise caution in using the GGA [or the local-density approximation (LDA)] single-particle spectra, since it suffers from its intrinsic selfinteraction error which results in band gap underestimation. ${ }^{30}$ This is a particularly important point in characterizing whether the levels closest to $E_{F}$, or the relevant channels for transmission, are the HOMO or the LUMO: If LUMO levels rather than HOMO levels lie closer to $E_{F}$, the qualitative reliability of the conclusion may be questioned. [For the systems considered in this work, we find that the HOMO levels lie closer to $E_{F}$ (Sec. III), so the conclusion is not affected by the GGA self-interaction errors.] A recent study also showed that the self-interaction error can possibly introduce a significant error for a weakly coupled molecular junction. ${ }^{31}$ To resolve this issue of DFT functionals in the transport calculation, it may help adopting new-generation self-interactionfree exchange-correlation energy functionals ${ }^{30,32}$ that were already shown to correct problems in the calculation of optical excitations. ${ }^{33}$

Related with this problem is that DFT is strictly valid only within the equilibrium and finite system setup. The KS Hamiltonian $H$, and the quantities derived thereafter, Green's functions $G$, self-energies $\Sigma$, and transmissions $T$, are in principle functions of the bias voltage $V$ in addition to the energy $E$. Self-consistent MGF calculations in which the finite-bias KS Hamiltonian for the molecule part is constructed are required for the accurate estimation of large-bias $I-V$ characteristics. However, even after this, the calculation is still inherently approximate as long as $H$ is calculated within the KS DFT formalism with the convectional exchange-correlation approximations. In this work, we carry out only the non-self-consistent MGF calculations based on zero-bias DFT calculations and restrict our interest to the low-bias regime where $T(E, V) \approx T(E, 0)$ should be a good approximation.

For now, the validity of approximations introduced in the calculation might be best assessed via comparison with accurate experiments which are beginning to appear. ${ }^{21-23}$ As will be described in Sec. III, for the case of single C6DT molecules probed by a small bias, we obtain a reasonable agreement with the experiment. One possible reason for such good comparison is that the transport properties of the present system under study, C6DT molecules strongly coupled to Au electrodes, are mainly determined via distribution of the HOMO levels along the electrode-molecule-electrode junction (that should be relatively well described by GGA or LDA) rather than that of the HOMO-LUMO gap (that should be inaccurately described by GGA or LDA). In any case, by such comparison, we believe that we are capturing the essential features of the systems considered in this work.

\section{APPLICATIONS: HEXANEDITHIOLATE MOLECULAR JUNCTIONS}

We now apply the developed methodology to study the effect of intermolecular interactions on the charge transport characteristics in C6DT devices. Extensive experimental studies were performed on the $n$-alkanethiol SAMs. ${ }^{4}$ In ambient conditions, $n$-alkanethiolates $(n \geq 6)$ self-assemble on a $\mathrm{Au}(111)$ surface to form a well-ordered monolayer with the $(\sqrt{3} \times \sqrt{3}) R 30^{\circ}$ packing density and the molecular tilt angle of $30^{\circ}$ from the surface normal. Various experimental techniques have found a secondary ordering to a $c(4 \sqrt{3}$ $\times 2 \sqrt{3}) R 30^{\circ}$ superlattice. The conductance properties of these SAMs have been extensively studied via various methods. ${ }^{34-36}$ 
(a)

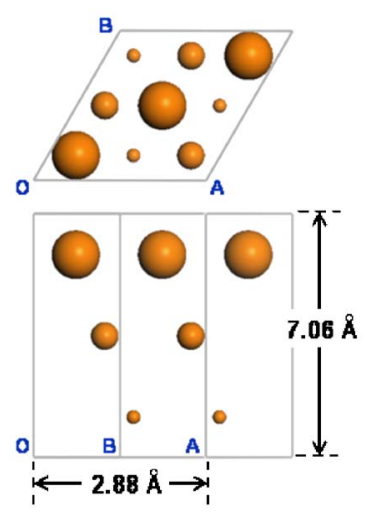

(b)

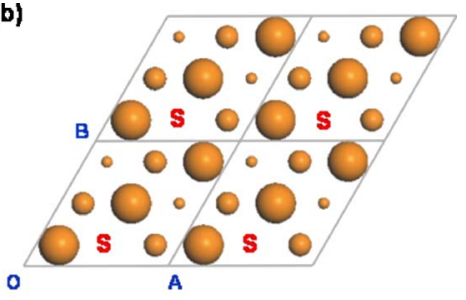

(c)

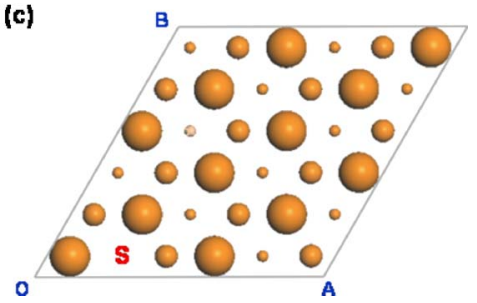

FIG. 2. (Color online) Top and side views of the (a) $(\sqrt{3}$ $\times \sqrt{3}) R 30^{\circ} \mathrm{Au}(111)$ cell corresponding to the bottom electrode. Top views of the (b) $2 \times 2(\sqrt{3} \times \sqrt{3}) R 30^{\circ} \mathrm{Au}(111)$ cell used for the monolayer calculations and (c) $(2 \sqrt{3} \times 2 \sqrt{3}) R 30^{\circ} \mathrm{Au}(111)$ supercell used for the single molecule calculations. In (b) and (c), S atoms that bridge the C6 molecular core to the fcc hollow site of the bottom electrode are shown together.

In addition to the experimental availability of wellordered SAMs and their conductance studies, our choice of C6DT molecule was largely motivated by the recent single molecular resistance measurements by $\mathrm{Xu}$ and Tao. ${ }^{21} \mathrm{By}$ repeatedly forming gold-molecule-gold junctions using STM, they have measured a very reliable single molecule resistance of $N$-alkanedithiol $(N=6,8$, and 10) molecules. For the case of octanedithiol, they have also obtained a consistent result by $\mathrm{Au}$-coated atomic force microscope (AFM) experiments. ${ }^{22}$ The clean experimental geometry in their experimental setup enabled unambiguous measurements of single-molecule resistance. For example, the measured resistance of the octanedithiol molecule was $51 \pm 5$ megaohms, which is an order of magnitude smaller than the probably best previous estimation of $900 \pm 50$ megaohms obtained in the experiment where a single molecule was bridged to the

Au-coated conducting AFM electrode through a gold nanoparticle. ${ }^{12}$

In this section, we will study the structures and charge transport characteristics of single and monolayer C6DT molecules sandwiched between flat $\mathrm{Au}(111)$ electrodes [Fig. 2(a)]. We first consider a single C6DT molecule stretched between $\mathrm{Au}(111)$ electrodes with the recent experiments ${ }^{21-23}$ in mind. A more realistic geometry of the electrodes corresponding to the experiments, in which the junctions were formed by pulling the STM or Au-coated AFM tip, would be a Au cluster or atomic wire bridging the bulk electrodes and the molecule as considered in Refs. 37 and 38. However, because one of our main objectives in this work is to compare charge transport characteristics of a single molecule and its monolayer counterpart, we will restrict ourselves to the flat electrode geometry where we can straightforwardly build monolayer models and corresponding single molecule models as schematically depicted in Figs. 2(b) and 2(c). In spite of the rather artificial flat electrode geometry, we obtain a computed conductance value in good agreement with the experimental data. We compare this conductance with that of the corresponding monolayer counterpart. Next, we consider the conductance of a relaxed C6DT monolayer and compare with that of the constituent single molecule. Using the four C6DT device models, we will assess the effect of intermolecular interactions within the monolayer and the molecular tilting on the C6DT device charge transport characteristics.

\section{A. Stretched single molecule and its monolayer counterpart}

Before calculating the device charge transport properties, we first carried out structural optimizations of a C6DT molecule stretched between two flat $\mathrm{Au}(111)$ electrodes. We placed two three-layer $(2 \sqrt{3} \times 2 \sqrt{3}) R 30^{\circ} \mathrm{Au}(111)$ cells [Fig. 2 (c)] mirror symmetrically as the bottom and top electrodes and optimized the structure of the molecule stretched between the electrodes (Fig. 3) within the PBE GGA while fixing all the Au atoms. The Au-molecule-Au slab was separated from its periodic images by $\sim 12 \AA$ of vacuum space

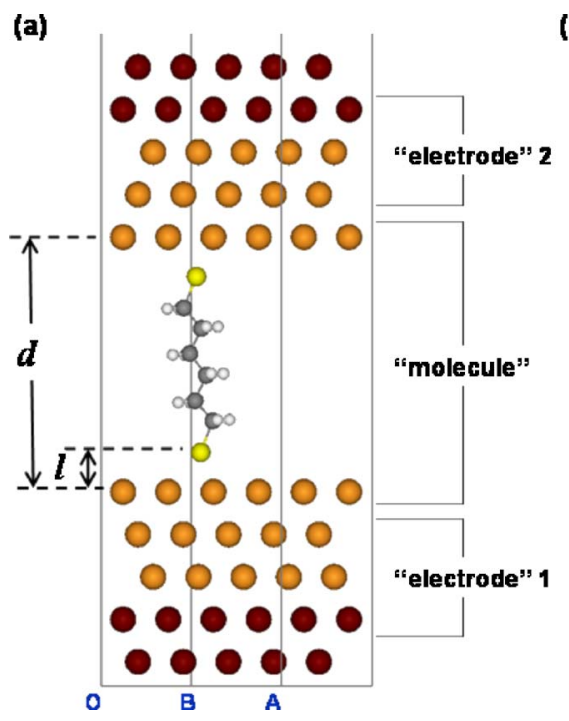

(b)

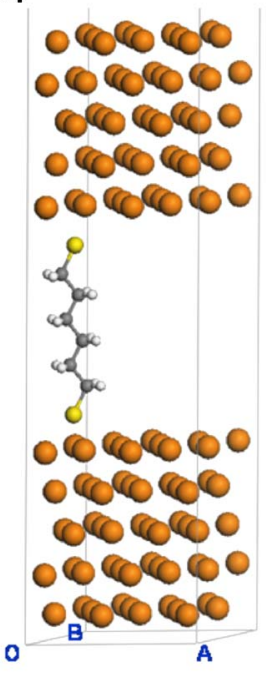

(c)

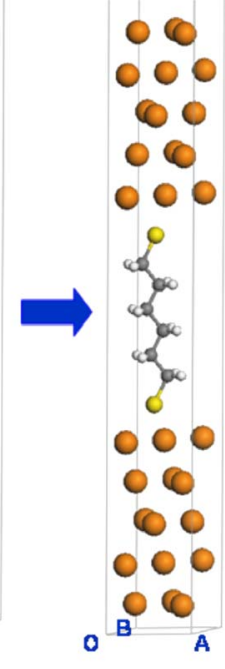

FIG. 3. (Color online) (a) Ball and stick representation of the stretched single C6DT device model. Three-layer Au(111) slabs [orange (gray)] were used for the energy minimization, and they were later augmented by another two $\mathrm{Au}(111)$ layers [magenta (dark gray)] for the calculation of the charge transport characteristics. Partitioning scheme of "electrodes" and "molecule" for the transmission calculations is also shown (see the text). The procedure of generating from (b) the single device model (c) the corresponding monolayer device model. 

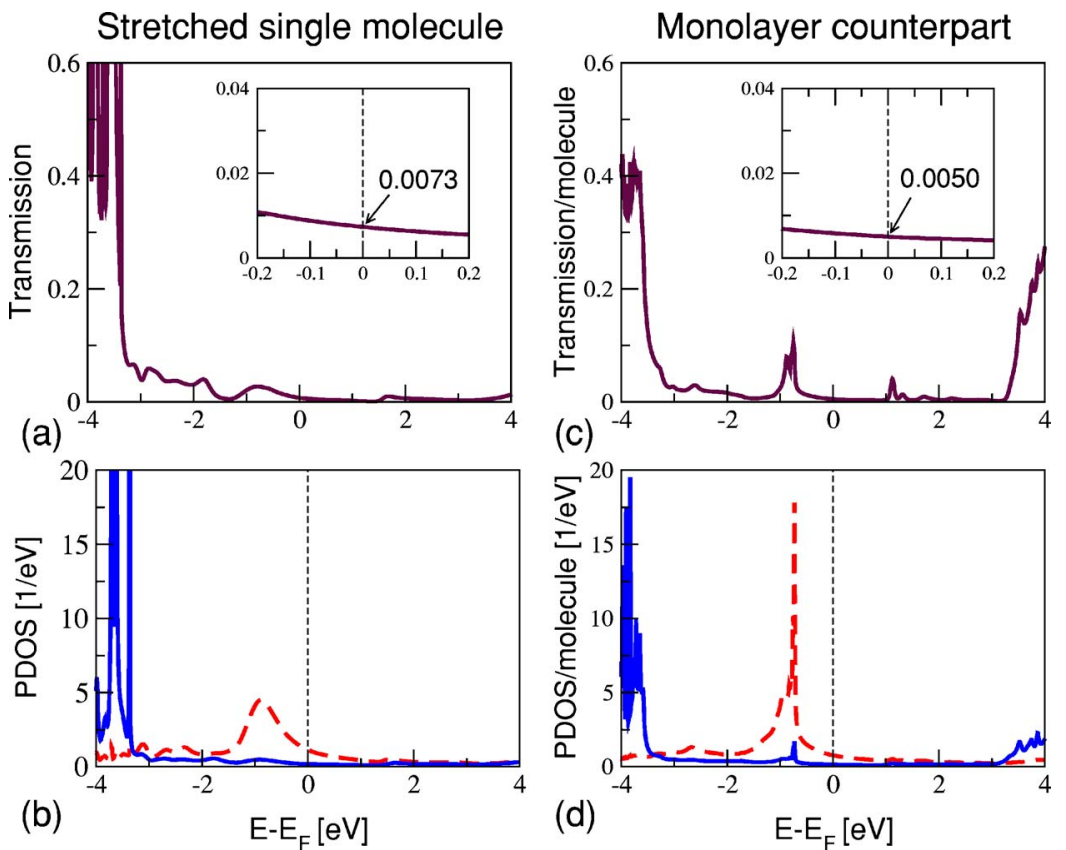

FIG. 4. (Color online) (a) Transmission function of a single stretched C6DT molecule and (b) its PDOS. (c) Transmission/molecule of the corresponding C6DT monolayer and (d) its PDOS. In (b) and (d), solid lines are molecular core (C6) projections and dashed lines are $\mathrm{S}$ bridge atoms (DT) projections. along the surface normal direction. Norm-conserving scalarrelativistic pseudopotentials were employed to remove the core electrons ${ }^{39,40}$ and orbitals were expanded in terms of the LCAO represented in the double- $\zeta$-plus-polarization quality Gaussian functions optimized for corresponding pseudopotentials. ${ }^{9}$ For the calculation of the exchange-correlation energy and potential and the Hartree potential, we adopted a uniform real-space grid with 0.3 bohr spacing. For reciprocal-space, a single $\Gamma \mathbf{k}_{\|}$point was sampled. Total energy was minimized until the maximum ionic forces were smaller than $25 \mathrm{meV} / \AA$.

An important aspect of the device structure is the location of the sulfur ("alligator clip") atoms with respect to the gold electrode surface layer atoms. For the alkane and phenyl molecules thiolate-attached to a $\mathrm{Au}(111)$ surface, we previously identified the fcc hollow site [Fig. 2(b)] as the energetically optimal position. ${ }^{10} \mathrm{Next}$, with the $\mathrm{S}$ atoms bridging the fcc hollow site of the $\mathrm{Au}(111)$ electrode, we allowed the two Au layers of the electrodes on the molecule $(\mathrm{S})$ side to relax and checked whether any significant reorganization of the molecule or $\mathrm{Au}$ atoms occurs. We repeated this process for progressively increasing electrode-electrode gap distances $d=13.7,13.9,14.1,14.3$, and $14.5 \AA$ (Fig. 3). It was found that at $d=14.3 \AA$ a noticeable protrusion of several gold atoms on one of the two $\mathrm{Au}$ surface layers develops. Increasing $d$ further to $14.5 \AA$, one of the three $\mathrm{Au}$ atoms in the perturbed layer closest to the $\mathrm{S}$ atom is pulled out of the $\mathrm{Au}$ surface plane. This result, reflecting the strong Au-S bonding, agrees with the Au wire formation and the subsequent $\mathrm{Au}-\mathrm{Au}$ bond breaking in the continued pulling of ethylthiolate molecule observed in a recent Car-Parrinello molecular dynamics study. ${ }^{37}$ As mentioned above, for the transmission calculations, we restrict ourselves to the flat electrode cases and we chose the case with the largest electrode-electrode gap distance, i.e., $d=14.1 \AA$ as the representative structure [Figs. 3(a) and 3(b)]. (Employing other choices, e.g., $d=14.1 \AA$ only result in minor quantitative changes in the results.) At this configuration, the distance between the $\mathrm{S}$ atom and the $\mathrm{Au}$ surface plane was $l$ $=2.19 \AA$.

Once the geometry was determined, we carried out MGF calculations to obtain the device charge transport characteristics. For the accuracy of the calculation, we padded each $\mathrm{Au}$ electrode slab with another two layers of $\mathrm{Au}$ electrode atoms (total of five layers of $\mathrm{Au}$ atoms for each electrode). Within the MGF formulation (Sec. II) we have decoupled the problem of computing the transmission function of a semiinfinite molecular junction into the DFT calculations of (i) extended molecule(s), i.e., molecule(s) plus a large part of bulk electrodes and (ii) two independent infinite electrodes, and the post-process coupling of these DFT output. In checking the convergence of the bulk electrode DFT calculations (ii) with respect to the $\mathbf{k}$ point sampling, we observed that the total energy converges and the "numerical" band gap approaches zero in the order of $10^{-3} \mathrm{eV}$ at a single $\mathbf{k}_{\|}$point shifted off from the $\Gamma$ point and four $\mathbf{k}_{\perp}$ points (Sec. II C 1). Due to the constraint of the $\mathbf{k}_{\|}$point matching between (i) and (ii) within our formulation (Sec. II B), we repeated the main device model calculation at the corresponding single $\mathbf{k}_{\|}$ point (shifted off from the $\Gamma$ point) as the input of the transmission calculation. We obtained the "numerical" HOMOLUMO gap of $<0.005 \mathrm{eV}$ in (i), which ensures the correct alignment of Fermi levels of (i) and (ii). Finally, we chose the molecule plus one layer of the Au electrode atoms as the "molecule" part of the device. This is because we have observed a rather noticeable deviation in the projected density of states (PDOS) of the Au surface layer from its bulk counterpart in our previous study of the BPDT devices. ${ }^{10}$ We used the next three layers of $\mathrm{Au}$ atoms (surface +1 -surface $+3 \mathrm{Au}$ layers) as the "electrode" part in the MGF calculations. This setup is schematically outlined in Fig. 3(a).

The transmission function of the device model based on the stretched single C6DT is shown in Fig. 4(a). Transmission value at $E_{F}, T\left(E=E_{F}\right)$, is 0.0073 , which can be compared with the conductance of $0.0012 G_{0}$ obtained in the 
STM experiment of $\mathrm{Xu}$ and Tao. ${ }^{21}$ The comparison is quite satisfactory considering that we have adopted a rather artificial flat electrode geometry. Our model should overestimate the conductance compared with the experimental situation, where the molecule is more likely connected to the electrodes through $\mathrm{Au}$ wires or clusters that are formed upon pulling of the STM tip. We analyzed the nature of the transmission channels via PDOS of S alligator clips (DT) and the remaining C6 molecular core [Fig. 4(b)]. It shows a DToriginated transmission at $\approx E_{F}-1.0 \mathrm{eV}$ and C6-originated transmissions at much further away from $E_{F}, \approx E_{F}-3.3 \mathrm{eV}$. Because of the DT states, the HOMO levels rather than the LUMO levels should be the more relevant transmission channels in this system, and this is consistent with the experimental conclusion. ${ }^{34}$ We previously found a similar behavior in the Au-BPDT-Au junctions where the DT states were also found to be located at $\approx E_{F}-1.0 \mathrm{eV},{ }^{10}$ and suspect that this might be a general conclusion for devices made by DT-bridging small molecules to Au electrodes. For example, employing ultraviolet photoemission spectroscopy, Zangmeister et al. determined the electronic levels of oligo(phenylene ethynylene)thiolate SAMs on Au and concluded that the HOMO is closer to $E_{F}$ than the LUMO. ${ }^{41}$ We in particular note that they located the Au-S level at $\approx E_{F}-1.0 \mathrm{eV}$ (See Fig. 5 of Ref. 41), which is in very good agreement with our calculations.

We now consider the monolayer devices and study the effect of intermolecular interaction on the charge transport properties accompanying the monolayer packing. To construct a monolayer device model, we reduced the surface unit cell from the $\mathrm{Au}(111)(2 \sqrt{3} \times 2 \sqrt{3}) R 30^{\circ}$ unit cell used in the "isolated" molecule device calculation to a $(\sqrt{3} \times \sqrt{3}) R 30^{\circ}$ unit cell. Pictorially, it corresponds going from Fig. 3(b) to Fig. 3(c) or Fig. 2(c) to Fig. 2(b). No further geometry optimization has been performed so that only the difference in the electronic degree of freedom is considered. The same calculational parameters as in the single-molecule case were adopted, except that $6 \times 6 \mathbf{k}_{\|}$points were sampled. As mentioned above, alkanethiolate molecules experimentally form a $c(4 \sqrt{3} \times 2 \sqrt{3}) R 30^{\circ}$ superstructure. Ignoring such superlattice structures, we overestimate the intermolecular interaction within the monolayer. We restrict ourselves to such simplification, because we find that the intermolecular interaction effect is negligible for our C6DT molecules (discussed below).

The transmission and PDOS of the stretched C6DT monolayer model are shown in Figs. 4(c) and 4(d), respectively. We indeed find effects of monolayer formation, such as the C6 band formation below $\approx E_{F}-3.5 \mathrm{eV}$ and above $\approx E_{F}$ $+3 \mathrm{eV}$, and further localization of DT PDOS near $E_{F}$ $-1 \mathrm{eV}$. However, for the energy range near $E_{F}$ that is relevant for the low-bias transport regime, we find only a small difference in their transmission values as shown in the insets of Figs. 4(a) and 4(c). For the SAM case, $T\left(E=E_{F}\right)$ is 0.0050 , which is slightly smaller than the value for the single molecule case (0.0073) due to the more localized $S$ levels near $E_{F}-1 \mathrm{eV}$. However, we expect this difference is negligible compared with the experimental indeterminacy and the computational approximations employed in the calculations (a)

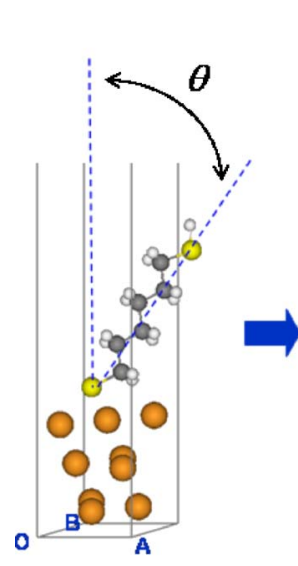

(b)

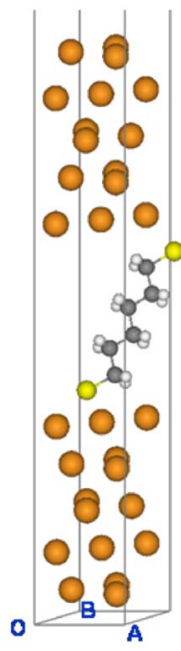

(c)

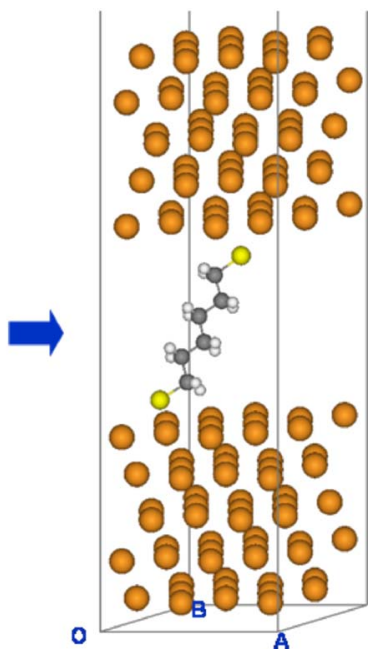

FIG. 5. (Color online) Ball and stick representation of (a) the optimized C6DT SAM, (b) the device model based on the SAM structure, and (c) the corresponding single molecule device model.

(Sec. II C). We thus conclude that for the C6DT monolayer stretched between $\mathrm{Au}(111)$ electrodes the coupling between molecular backbones does not affect the charge transport along the surface normal direction in the low-bias transport regime.

\section{B. Self-assembled monolayer and its single molecule counterpart}

For the devices based on stretched molecules studied in Sec. III A, where the direction of the charge flow is parallel to the alignment of the molecular backbone, it might be regarded as natural to find negligible intermolecular interaction effects on the charge transport. In this section, we will consider the case of self-assembled monolayers in which molecules are tilted away from the electrode surface normal direction. In this case, it is not clear from the outset whether we will find a similar behavior. We will show that, even with the molecule tilting, the C6DT electrical transport property at the low-bias regime is not significantly modified by the intermolecular interactions.

We first optimized the structure of the SAM model based on the three-layer $\operatorname{Au}(111)(\sqrt{3} \times \sqrt{3}) R 30^{\circ}$ substrate [Fig. 5(a)] using PBE GGA. Reciprocal-space $3 \times 3 \mathbf{k}_{\|}$points were sampled, and other calculational parameters were taken as in the structural study of the stretched single molecule case. As discussed in Sec. III A, the $(\sqrt{3} \times \sqrt{3}) R 30^{\circ}$ unit cell should overestimate the intermolecular interaction effects compared with the experiments, which identified the $c(4 \sqrt{3}$ $\times 2 \sqrt{3}) R 30^{\circ}$ superlattice structure. All Au atoms were fixed during the optimization. We have previously found that allowing the relaxation of the surface Au atoms results in only minor structural changes and has an especially negligible effect on the device charge transport characteristics of thiolbased SAMs. ${ }^{10}$ The tilt angle from the surface normal of the optimized molecules was $\theta=31.9^{\circ}$ and the distance of the 
Single molecule counterpart
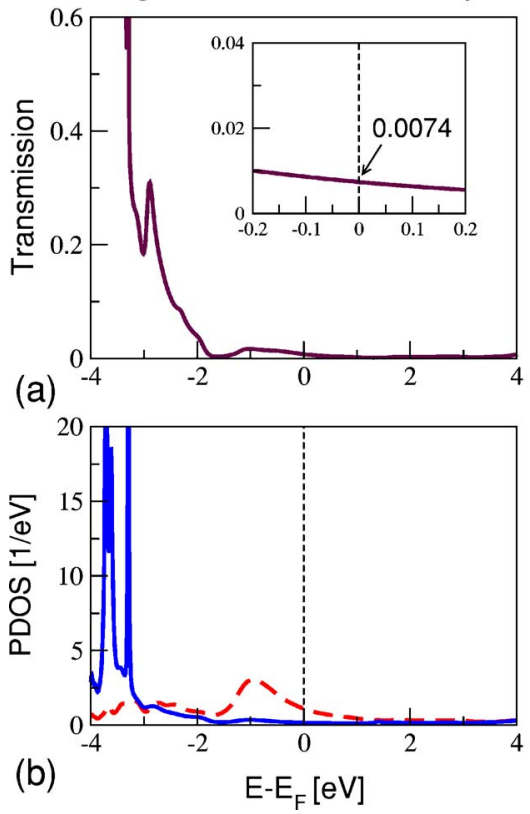
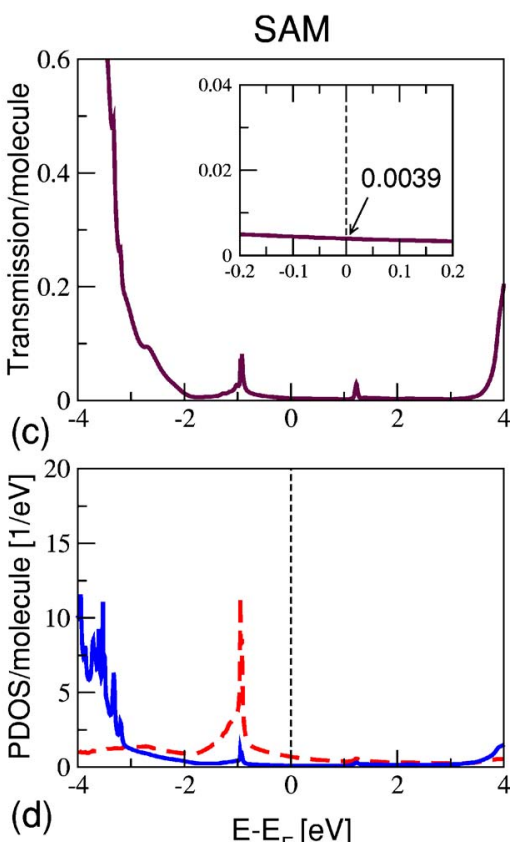

FIG. 6. (Color online) (c) Transmission/ molecule of the optimized C6DT monolayer and (d) its PDOS. (a) Transmission function of the corresponding single C6DT molecule and (b) its PDOS. In (b) and (d), solid lines are C6 projections, and dashed lines are DT projections. bottom $\mathrm{S}$ atom from the top atomic layer of the $\mathrm{Au}$ substrate was $l=2.16 \AA$.

We next constructed a device model by removing the $\mathrm{H}$ atom of the top thiol and capping the optimized SAM structure with another three-atomic-layer $\mathrm{Au}(111)$ slab as the top electrode. This top $\mathrm{Au}(111)$ slab was placed such that the device model possesses mirror symmetry with respect to the center of the molecule: The top S atom bridges the C6 molecular core to the fcc site of the top electrode and the distance between the $\mathrm{S}$ atom and the top Au electrode surface layer is $l=2.16 \AA$. The electrode-electrode gap distance was $d=11.29 \AA$. With this device model, we once more performed geometry optimization of the molecule while fixing all the $\mathrm{Au}$ atoms. This resulted in a minimal structural change of the molecule: $\theta=31.3^{\circ}$ and $l=2.15 \AA$. As in Sec. III A, after structural optimizations, we padded each electrode with another two $\mathrm{Au}(111)$ layers (total of five layers of $\mathrm{Au}$ atoms for each electrode) for the final transmission calculation [Fig. 5(b)]. To analyze the intermolecular interaction effect on this optimized SAM, we constructed a corresponding single device model by making a $2 \times 2$ supercell of the monolayer device model and removing three of the four C6DT molecules [Fig. 5(c)].

Transmission functions of the single and SAM C6DT device models are shown in Figs. 6(a) and 6(c), respectively. Corresponding PDOS data are presented in Figs. 6(b) and 6(d). Comparing the two, we find monolayer packing effects similar to those for the stretched molecule case (Sec. III A) such as the $\mathrm{C} 6$ band formation below $E_{F}-3 \mathrm{eV}$ and above $E_{F}+3 \mathrm{eV}$ and the localization of DT PDOS near $E_{F}-1 \mathrm{eV}$. This results in a slightly smaller $T\left(E=E_{F}\right)=0.0039$ of the SAM model compared with that of the single molecule model, 0.0074. In general, however, the two transmissions show very similar features near $E_{F}$, indicating negligible effect of SAM formation on the charge transport properties at the low-bias transport regime.

We next focus on the effect of molecule tilting by comparing Figs. 6 and 4. First, in terms of PDOS, we find a noticeable change (stronger band formation) only for the SAM case below $E_{F}-3 \mathrm{eV}$ [Figs. 4(d) and 6(d)]. For transmissions, we find a noticeable enhancement below $E_{F}$ $-2 \mathrm{eV}$ for both tilted single molecule and SAM device models [Figs. 6(a) and 6(c)] compared with the corresponding device models based on stretched molecules [Figs. 4(a) and 4(c), respectively]. However, these changes arise only for the energy range far away from $E_{F}$ that is relevant for the highbias $(>\sim 4 \mathrm{~V})$ regime $I-V$ characteristics. The transmission characteristics relevant for the low-bias regime (energy range close to $E_{F}$ ) show only a negligible difference with the molecule tilting and SAM formation as shown in the $I-V$ data of Fig. 7, calculated according to Eq. (1) with $\mu_{1}=E_{F}-0.5 \mathrm{eV}$ and $\mu_{1}=E_{F}+0.5 \mathrm{eV}$. From this, we conclude that C6DT pos-

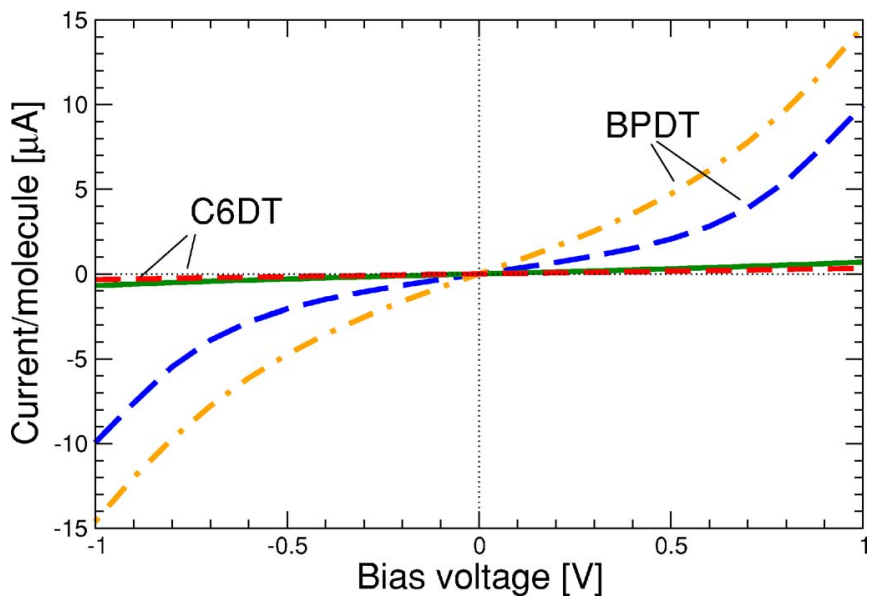

FIG. 7. (Color online) Per molecule $I-V$ characteristics of devices based on the stretched single C6DT molecule (solid lines) and optimized C6DT SAM (dashed lines). For comparison, per molecule $I-V$ characteristics of herringbone-packing $\theta \approx 15^{\circ}$ BPDT SAM device (long dashed lines) and parallel-packing $\theta \approx 30^{\circ}$ BPDT SAM device (dot-dashed lines) from Ref. 10 are shown together. 
sesses a robust charge transport property and will be a good device component. In Fig. 7, because the four $I-V$ curves showed very similar behavior, we present the data for only two models (stretched single molecule and optimized SAM cases) that showed the biggest difference. To emphasize the insignificance of this difference, together we present the $I-V$ curves of two BPDT SAM device models from Ref. 10. We will discuss the differences in more detail in Sec. IV.

\section{SUMMARY AND DISCUSSIONS: SATURATED VS $\pi$-CONJUGATED MOLECULES}

Compared with single isolated molecules, molecules within a monolayer can possibly assume more robust molecular structures due to the intermolecular interactions. This aspect might be utilized as a device control mechanism in molecular electronics. In this article, we reported on the development of a first-principles computation tool to study the charge transport properties of monolayer molecular electronic devices and its application to the C6DT wires. By studying an isolated C6DT single molecule, we first established a good quantitative agreement of our calculation with the recent single molecule resistance measurements. ${ }^{21}$ Considering that a large quantitative discrepancy between theoretical calculations and experimental data has been one of the most unsatisfactory aspects of the field of molecular electronics, we believe that this finding is encouraging. Next, we studied the charge conductance of monolayers and compared it with that of single molecule counterparts. We concluded that the monolayer packing and the molecule tilting have only a minor effect on the C6DT transmission characteristics in the low-bias regime. Taking into account their wellcharacterized SAM structure as well, we concluded that the $n$-alkanedithiolates would be a good (insulating) molecular electronics component.

We close the paper by comparing these findings with those for the devices based on $\pi$-conjugated BPDT molecules which we previously considered. ${ }^{10}$ The electrodeelectrode gap distance of the BPDT device models was 13.6 14.7 A, comparable to that of the present C6DT device models. Note that, although we did not directly compare the single molecule conductance and the SAM conductance for BPDT devices as in the current work, we can still assess the effects of molecule tilting and monolayer packing on the BPDT device $I-V$ characteristics via comparing the conductance of SAMs with different molecule tilt angles and packing modes. We first found that the potential energy surface as a function of the BPDT tilt angle is very flat. We thus concluded that the BPDT SAMs should not be well-ordered, in agreement with the experimental observation. ${ }^{42,43} \mathrm{We}$ in ad- dition observed a sensitive dependence of BPDT transmission near $E_{F}$ or low-bias regime $I-V$ characteristics on the molecule tilting. The origin of this behavior was traced to the HOMO-LUMO gap of the $\pi$-conjugated BP molecular core, which is much smaller than that of the saturated C6 core, and the resulting strong hybridization of BP levels with DT levels. As discussed in Sec. III A, we observed DT levels at $\approx E_{F}-1 \mathrm{eV}$ in BPDT devices as in C6DT devices. However, unlike the C6DT case that shows negligible C6 PDOS near $E_{F}$ [Figs. 3(b), 3(d), 5(b), and 5(d)], a relatively large BP PDOS (yet smaller than DT PDOS) was found between $\approx E_{F}-1$ and $E_{F}$ (Fig. 7 of Ref. 10). Because of the structural indeterminacy and sensitive dependence of the $I-V$ characteristics on the conformational variations, we concluded that BPDT molecules are a poor device candidate. To contrast the C6DT and BPDT cases and provide a quantitative measure of these conclusions, we reproduced the $I-V$ curves of the herringbone-packing $\theta \approx 15^{\circ}$ BPDT SAM and parallelpacking $\theta \approx 30^{\circ}$ BPDT SAM devices (Fig. 9 of Ref. 10) in Fig. 7.

We finally note that our comparison of C6DT and BPDT conductances is in line with the experimental characterization of $n$-alkanedithiol ${ }^{21,22}$ and monophenyldi(methane) thiol $^{23}$ by the Tao group: Compared with the saturated $n$-alkanedithiol molecules, conductance measurements of $\pi$-conjugated benzenedithiol and benzenedimethanethiol junctions were found to have a lower device yield and broader conductance histograms, or a less well-defined single molecule conductance value. This can be explained by our finding that the $\pi$-conjugated molecular cores connected to metal electrodes via thiol bridges have less stable structures and bigger conductance fluctuations compared with the saturated molecular counterparts.

\section{ACKNOWLEDGMENTS}

Y.-H.K. was supported by the Korea Research Foundation Grant funded by the Korean Government (MOEHRD) (Grant No. KRF-2005-041-C00125), in which main calculations were performed by using the supercomputing resource of the Korea Institute of Science and Technology (KISTI). J. T.-K. and W.A.G. III were supported by NSF Nanotechnology and Interdisciplinary Research Initiative and MARCO Focus Centers on Functional Engineered NanoArchitectonics. W.A.G. III was also partially supported by Intel Components Research. The facilities of the MSC were supported by ONR-DURIP, ARO-DURIP, NSF-MRI, and the Beckman Institute. Sandia is a multiprogram laboratory operated by Sandia Corporation, a Lockheed Martin Company, for the US DOE under Contract No. DE-AC04-94AL85000.

\footnotetext{
*Author to whom correspondence should be addressed. Electronic address: yhkim@kias.re.kr

†Electronic address: wag@wag.caltech.edu

${ }^{1}$ J. R. Heath and M. A. Ratner, Phys. Today 56, 43 (2003).
}

${ }^{2}$ R. L. McCreery, Chem. Mater. 16, 4477 (2004).

${ }^{3}$ R. Smith, P. Lewis, and P. S. Weiss, Prog. Surf. Sci. 75, 1 (2004).

${ }^{4}$ J. C. Love, L. A. Estroff, J. K. Kriebel, R. G. Nuzzo, and G. M. Whitesides, Chem. Rev. (Washington, D.C.) 105, 1103 (2005). 
${ }^{5}$ P. M. Mendes, A. H. Flood, and J. F. Stoddart, Appl. Phys. 80, 1197 (2005).

${ }^{6}$ J. G. Kushmerick, J. Naciri, J. C. Yang, and R. Shashidhar, Nano Lett. 3, 897 (2003).

${ }^{7}$ A. S. Blum, J. G. Kushmerick, S. K. Pollack, J. C. Yang, M. Moore, J. Naciri, R. Shashidhar, and B. R. Ratna, J. Phys. Chem. B 108, 18124 (2004).

${ }^{8}$ Y. Selzer, L. Cai, M. A. Cabassi, Y. Yao, J. M. Tour, T. S. Mayer, and D. L. Allara, Nano Lett. 5, 61 (2005).

${ }^{9}$ P. A. Schultz, Sandia National Laboratories, http://dft.sandia.gov/ Quest

${ }^{10}$ Y.-H. Kim, S. S. Jang, and W. A. Goddard III, J. Chem. Phys. 122, 244703 (2005).

${ }^{11}$ Y.-H. Kim, S. S. Jang, Y. H. Jang, and W. A. Goddard III, Phys. Rev. Lett. 94, 156801 (2005); Y.-H. Kim, S. S. Jang, and W. A. Goddard III, Appl. Phys. Lett. 88, 163112 (2006).

${ }^{12}$ X. D. Cui, A. Primak, X. Zarate, J. Tomfohr, O. F. Sankey, A. L. Moore, T. A. Moore, D. Gust, G. Harris, and S. M. Lindsay, Science 294, 571 (2001).

${ }^{13}$ P. S. Damle, A. W. Ghosh, and S. Datta, Phys. Rev. B 64, 201403(R) (2001); Chem. Phys. 281, 171 (2002).

${ }^{14}$ Y. Xue, S. Datta, and M. A. Ratner, J. Chem. Phys. 115, 4292 (2001); Y. Xue and M. A. Ratner, Phys. Rev. B 68, 115406 (2003).

${ }^{15}$ J. J. Palacios, A. J. Pérez-Jiménez, E. Louis, E. SanFabián, and J. A. Vergés, Phys. Rev. B 66, 035322 (2002).

${ }^{16}$ J. Taylor, H. Guo, and J. Wang, Phys. Rev. B 63, 245407 (2001).

${ }^{17}$ M. Brandbyge, J.-L. Mozos, P. Ordejón, J. Taylor, and K. Stokbro, Phys. Rev. B 65, 165401 (2002).

${ }^{18}$ S.-H. Ke, H. U. Baranger, and W. Yang, Phys. Rev. B 70, 085410 (2004)

${ }^{19}$ A. R. Rocha, V. M. García-Suárez, S. W. Bailey, C. J. Lambert, J. Ferrer, and S. Sanvito, Nat. Mater. 4, 335 (2005).

${ }^{20}$ W. Lu, V. Meunier, and J. Bernholc, Phys. Rev. Lett. 95, 206805 (2005).

${ }^{21}$ B. Xu and N. J. Tao, Science 301, 1221 (2003).

${ }^{22}$ B. Xu, X. Xiao, and N. J. Tao, J. Am. Chem. Soc. 125, 16164 (2003)
${ }^{23}$ X. Xiao, B. Xu, and N. J. Tao, Nano Lett. 4, 267 (2004).

${ }^{24}$ S. Datta, Quantum Transport: Atom to Transistor (Cambridge University Press, Cambridge, UK, 2005).

${ }^{25}$ H. Haug and A.-P. Jahuo, Quantum Kinetics in Transport and Optics of Semiconductors (Springer, Berlin, 1996).

${ }^{26}$ A. R. Williams, P. J. Feibelman, and N. D. Lang, Phys. Rev. B 26, 5433 (1982).

${ }^{27}$ Y. Meir and N. S. Wingreen, Phys. Rev. Lett. 68, 2512 (1992).

${ }^{28}$ J. P. Perdew, K. Burke, and M. Ernzerhof, Phys. Rev. Lett. 77, 3865 (1996).

${ }^{29}$ W. Kohn, Rev. Mod. Phys. 71, 1253 (1999).

${ }^{30}$ M. Städele, J. A. Majewski, P. Vogl, and A. Görling, Phys. Rev. Lett. 79, 2089 (1997).

${ }^{31}$ C. Toher, A. Filippetti, S. Sanvito, and K. Burke, Phys. Rev. Lett. 95, 146402 (2005).

${ }^{32}$ Y.-H. Kim, M. Städele, and R. M. Martin, Phys. Rev. A 60, 3633 (1999).

${ }^{33}$ Y.-H. Kim and A. Görling, Phys. Rev. Lett. 89, 096402 (2002).

${ }^{34}$ J. M. Beebe, V. B. Engelkes, L. L. Miller, and C. D. Frisbie, J. Am. Chem. Soc. 124, 11268 (2002); V. B. Engelkes, J. M. Beebe, and C. D. Frisbie, ibid. 126, 14287 (2004).

${ }^{35}$ J. G. Kushmerick, D. B. Holt, S. K. Pollack, M. A. Ratner, J. C. Yang, T. L. Schull, J. Naciri, M. H. Moore, and R. Shashihar, J. Am. Chem. Soc. 124, 10654 (2002).

${ }^{36}$ W. Wang, T. Lee, and M. A. Reed, Phys. Rev. B 68, 035416 (2003).

${ }^{37}$ D. Kruger, H. Fuchs, R. Rousseau, D. Marx, and M. Parrinello, Phys. Rev. Lett. 89, 186402 (2002).

${ }^{38}$ P. Vélez, S. A. Dassie, and E. P. M. Leiva, Phys. Rev. Lett. 95, 045503 (2005).

${ }^{39}$ D. R. Hamann, Phys. Rev. B 40, 2980 (1989).

${ }^{40}$ N. Troullier and J. L. Martins, Phys. Rev. B 43, 1993 (1991).

${ }^{41}$ C. D. Zangmeister, S. W. Robey, R. D. van Zee, Y. Yao, and J. M. Tour, J. Phys. Chem. B 108, 16187 (2004).

${ }^{42}$ T. Y. B. Leung, P. Schwartz, G. Scoles, F. Schreiber, and A. Ulman, Surf. Sci. 458, 34 (2000).

${ }^{43}$ W. Azzam, C. Fuxen, A. Birkner, H.-T. Rong, M. Buck, and C. Wöll, Langmuir 19, 4958 (2003). 